\title{
Automaton-on-Tag: An Approach for an RFID-driven production control with Mealy machines stored on an RFID tag
}

\author{
Timo Busert, Aljosha Köcher, Robert Julius, Alexander Fay \\ Helmut Schmidt University / University of the Federal Armed Forces Hamburg, Institute of \\ Automation Technology, Holstenhofweg 85, 22043 Hamburg (Germany) \\ timo.busert@hsu-hh.de, aljosha.koecher@hsu-hh.de, \\ robert.julius@hsu-hh.de, alexander.fay@hsu-hh.de
}

\begin{abstract}
In this paper, we present an approach to how to store production plans directly on an RFID tag in the form of an automaton. Based on a modular manufacturing system, this enables manufacturing systems to become more flexible and changeable and, in addition, reduces the engineering effort for adaptation in an existing plant. The connection between different production modules is implemented via carriers and a Mealy machine that is stored on an RFID tag. This machine's states represent the production steps of the product on the carrier.
\end{abstract}

Keywords: Data-on-Tag, Automaton-on-Tag, CPS, modular manufacturing system, SOA, Mealy machine

\section{Introduction}

Production plants are facing a dynamic environment. The emerging demand for individualized products (down to one-piece production) to similar costs compared to bulk products and competitive in quality, price and availability results in changing challenges during the lifetime of a plant [1]. This is especially for manufacturing systems with a high degree of automation challenging. Nowadays these systems are highly complex so that they are often less flexible or changeable. Due to their complexity, adaptions and reconfigurations can be very time consuming. Flexibility and changeability are two requirements to meet the challenges of the dynamic environment. Concepts, aspects and technologies emerging with the buzzword "Industrie 4.0" are enablers to reduce complexity within a production system, whereupon flexibility and changeability can be gained. A central concept of Industrie 4.0 are Cyber-Physical Systems (CPS). CPS are smart machines, warehouse systems, resources or products that are able to communicate with each other and control each other [2]. Products become due to CPS clearly identifiable, are localizable, know their production history, their current production state and alternatives to get to their target state [2]. Another aspect of Industrie 4.0 is that storing and processing of information should be close to the physical location where the information origins from or where the information is needed [3]. This results

adfa, p. 1, 2011.

(c) Springer-Verlag Berlin Heidelberg 2011 
in a decentrally organized, robust and flexible process of data processing [3]. In this paper, we describe an approach to how to reduce complexity and gain more flexibility in a manufacturing system by the application of Industrie 4.0 approaches. We use RFID tags to let objects, i.e. products, become CPS and store the whole workflow in the form of a Mealy machine on it, thus guiding the product through the production process. This paper is structured as follows: After a short introduction, explaining the problem, motivation and a short introduction to a possible solution, in Section 2 the background and related work is discussed. Section 3 presents a possibility of a decentral data management. In Section 4, we demonstrate the concept Automaton-on-Tag, whose implementation is demonstrated in Section 5. This paper concludes with a summary and an outlook towards future research topics.

\section{Background and Related Work}

\subsection{Typical information flow in automated manufacturing systems}

Nowadays the information flow in a manufacturing system is often centralized and strictly hierarchically structured. Several IT-systems are used to perform different tasks and communicate with each other across different hierarchy levels.

Initially a production order will be registered in an ERP-System. This order will be handed over to the MES that is in charge of the production process and handles all following decisions, including scheduling of orders and assigning of orders to machines [1]. Via Supervisory Control and Data Acquisition (SCADA), manual interventions into the manufacturing process are possible and a representation of the current state of the manufacturing system is visible. PLCs control the physical manufacturing process via sensors and actors. This structure leads to high complexity of the manufacturing system and within the procedure of controlling and scheduling of the production. This causes that even simple changes in a running production process will effect adaptations in different IT-Systems.

\subsection{Modular Plants and SOA}

An approach for a reduction of the complexity within a manufacturing system is seen in decentralization and modularization, so that single control tasks are spatially and chronologically separated and that these tasks can be executed autonomously by the modules [4]. This approach leads to a dissolving of the strictly hierarchically structured automation pyramid, shown in figure 1 (left). For that, each machine or different machines related to each other will be capsulated in a module. Each module features one or several services according to its functionalities. This leads to a Service-Oriented Architecture (SOA). SOA is a concept originating from the IT, based on services. Each service represents a certain functionality and provides only this functionality to other services [5]. The implementation of the functionality inside of the service is not visible from outside the service [5]. In the SOA concept all services are loosely coupled, so they operate independently from each other, their interactions are stateless, asynchronous and not context-related [6]. Applications and production workflows in SOA are a 
composition of different services, which together fulfill a mission [5]. Due to the loose coupling of the services, manufacturing systems become more flexible and changeable.

\section{Decentral Data Management in Modular Plants}

\subsection{A possible Structure of the Automation Pyramid in a modular Plant with RFID-driven production control}

In a modular manufacturing system each module offers at least one service. Due to this, the rigid automation pyramid gets softened. We propose an approach for an RFIDdriven production control. All related information to a product will be directly linked to the product or a carrier, transporting the product through the manufacturing system. The RFID tag contains all relevant information to control the production process. Thus, it is also able to realize the communication between the modules and the IT-Systems.

The right side of figure 1 shows a way to divide the automation pyramid and the communication structure when using a modularized manufacturing system and carriers to realize the communication. While some SCADA functionalities might be integrated into the MES, a typical SCADA system cannot be used if a plant's modules are only connected by carriers.

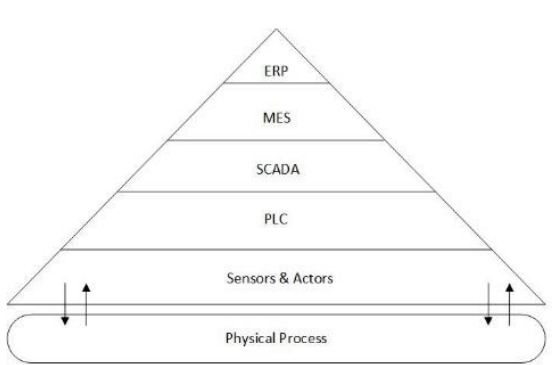

Fig. 1. Typical Automation Pyramid (left) \& modularized Automation Pyramid

(right)

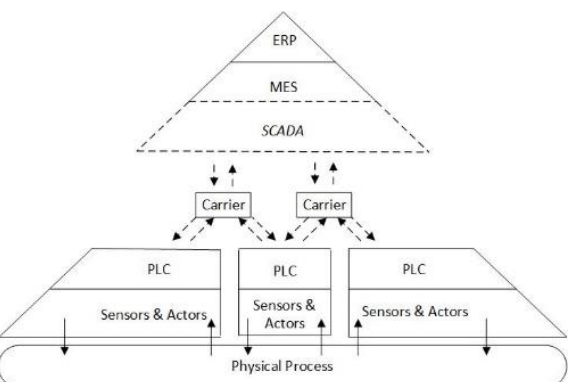

\subsection{Requirements and Existing Work for a decentral Data Management}

In most use cases, RFID tags are only used as an identifier for objects, storing just a unique identifier, despite the fact that the tag has enough capacity to store object related information directly to it [7]. Often object related data are stored in a backend system [7]. This approach is called "data-on-network" [7]. The approach data-on-network brings several disadvantages, like a high complexity and the circumstance that all modules are dependent on a constant network connection to a master database. A possibility to solve these problems is storing all the objet relevant data directly on the tag, as mentioned above. This approach is called "data-on-tag" and leads to a decentral data management [7]. [8] define some rules and requirements for the implementation of a data- 
on-tag approach, which have to be considered to develop a sustainable approach for a decentral data management:

- Implementation of a low cost memory, to consider the case, that the memory is connected to the product for the whole life cycle. A consequence of this is the application of a method, requiring a small amount of storage space.

- Sufficient product storage space, to store all manufacturing information.

- Flexibility of the data structure, so that the data structure is easy adoptable to new conditions.

- Expandability of the memory, to provide the option to extend the related information to an external storage space, if the memory does not remain on the product.

- Fast read and write access to the memory to ensure an efficient production process.

A further requirement for a sustainable approach of a decentral data management is the consideration of a possible flexibility within a production process. The description model of the production workflow on the RFID tag has to be able to consider different possibilities within the production process, like loops, alternative branches or fixed steps.

An existing approach for a decentralized data management is presented in [9]. Based on the idea of using agents for controlling the production process, the approach is called Agent-on-Tag. The core aspect is to store a whole agent on a RFID tag. Every time the RFID tag is connected to a module or a machine, the executables will be executed and the runtime variables will be updated. This is only applicable for RFID systems and tags with a huge storage space and a fast speed for reading and writing the RFID tag [9]. Thus, it does not fulfill the requirements mentioned above.

[8] present an approach for a decentral data management in a modular plant. They store the current manufacturing state on the tag. Every time the RFID tag is connected to a module, the module compares the current manufacturing state of the product with the requirements to execute the manufacturing service of the module. With this method, they are not able to consider different possibilities within the production process, like loops and alternatives.

\section{Automaton-on-Tag}

\subsection{Selection of a Description Model}

To meet all requirements defined above, we use three types of information, stored on the RFID tag. First, there are data needed for identification and description of the related product. Second, there are data needed, for controlling the production process and third, there could be a need for production related data. These production related data could be used to reconstruct the production process ex post. The focus in this paper is on the second type of data, the data for controlling the production process. A possible method to meet the described requirements above is to describe the production process in form of an automaton on the RFID tag. Automatons are mathematical models to describe 
discrete event processes and systems and consist at least of different states and edges, connecting the states [10]. The state of the automaton is exactly defined and the automaton can only be in one of a finite number of states at any given time. In this context, a state represents a fulfilled service of a module, so that the current state is the last executed production step. Through the edges, the states are getting related to each other. If there is a connection between two states, a change from the current state to the other is possible. Thus, the edges define possible paths through a network of states, describing the sequence in which the production steps has to be fulfilled. Since in a manufacturing process the transitions from one state to another are related to certain preconditions, for instance Step A has to be fulfilled before Step B, the transitions have to be linked to some conditions, which have to be fulfilled before a change from one state to another is permitted. In this context these conditions are linked to the edges. In manufacturing systems modules might not only offer fixed services, but also services that provide some options. An example for this circumstance could be the fictional service painting. This service can be executed with different colors. So there is a need for parameters to define the way how each module executes its services. These parameters can also be linked to the edges. A specification of an automaton, enabling the consideration of both these requirements, is the Mealy machine. In a Mealy machine, the transition from one state to another depends on an input [10]. An output is determined by its current state and the current input [10]. The input can be interpreted as a condition considering the production history. Depending on the current state and the condition, the output specifies the services to be executed.

\subsection{Data Structure on the Tag}

To store the Mealy machine on an RFID tag, we use three data components. 1. current state, representing the last executed production step, 2. production history, representing all the executed services and 3 . edges,. The current state expresses the last executed step in form of a number representing the fulfilled service. This data component is necessary to define the current position within the Mealy machine. The production history is realized as an array, containing the number of invocations of each service. Edges contain the source and the target state, the condition that needs to be fulfilled to choose this edge and a parameter, specifying the execution of the services. Figure 2 demonstrates the declaration of two user defined types that are used by each module's controller to interpret the RFID tag data as a Mealy machine. The first type contains the current state, the production progress and an array of edges. These edges are defined by the second structure. While we use a simple integer as an identification number, the Electronic Product Code (EPC) might be more suited for industrial use. The EPC is a universal identifier, providing a unique identity assigned to physical objects, unit loads, locations, or other identifiable entities. Additionally we extended the edges with time stamps. This enables a chronological reconstruction of the production history and allows the monitoring of each module's processing time and every products lead time. 


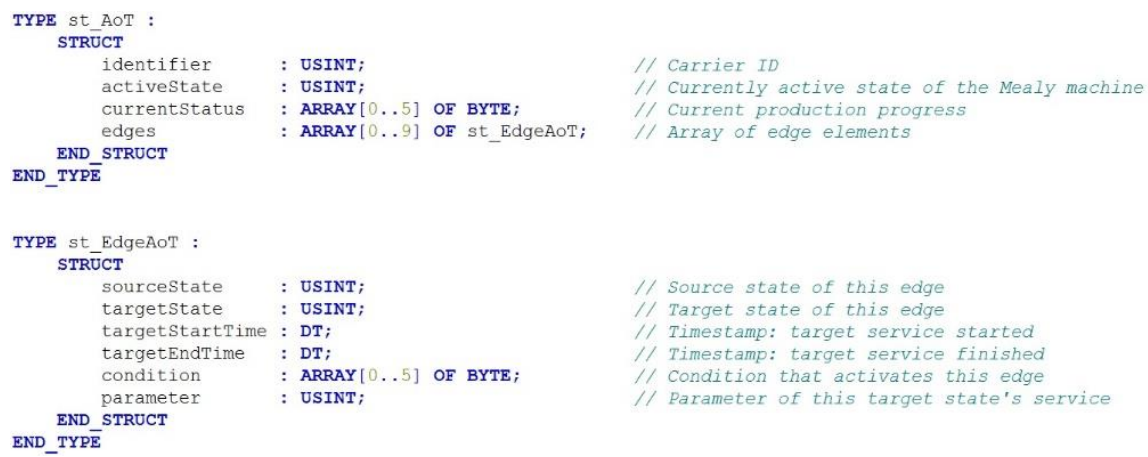

Fig. 2. Data Structure of Automaton-on-Tag

\subsection{Processing of the Data}

Through the described data structure, the complete Mealy machine becomes representable on the RFID tag. As RFID tags are only able to communicate passively the active part of the communication has to be fulfilled by the module. Every time an RFID tag comes to a module, the module has to read the data, process them, update them, and write them on the RFID tag. This procedure happens as follows: if an RFID tag comes into the range of the RFID reader of a module, the module itself starts to read the information on the tag. Beginning with the first edge, the PLC compares (a) if the current state, stored on the RFID tag is equal to the source state of the edge, (b) if the target state of the edge is equal to the state the module is representing and (c) if the condition relating to the edge is equal to the production history. If an edge fulfills these three conditions, this edge's parameter will be transmitted and the target service's start time will be set. After executing the service, its end time will be updated. This is followed by a physical transport to the next module, where the procedure happens again. If the condition of an edge is not fulfilled, the PLC checks, if the last edge is reached. If this is not the case, the next edge will be selected and the procedure happens again. If the last edge is reached, there was no intention to execute the service of the corresponding module within the production flow of the product and the product will be transported to the next module. Figure 3 describes the sequence of the data processing in form of a flowchart. This procedure and data format enables the consideration of loops and alternatives within the production workflow. Loops are realized through the conditions of the edges. Equivalent alternatives are realized through equal source states and equal conditions of the edge, but different target states. Due to the interaction of the components and the dynamic data structure the object carrying the RFID tag becomes a CPS, which is able to navigate itself through the production process and which is able to communicate with the different modules. 


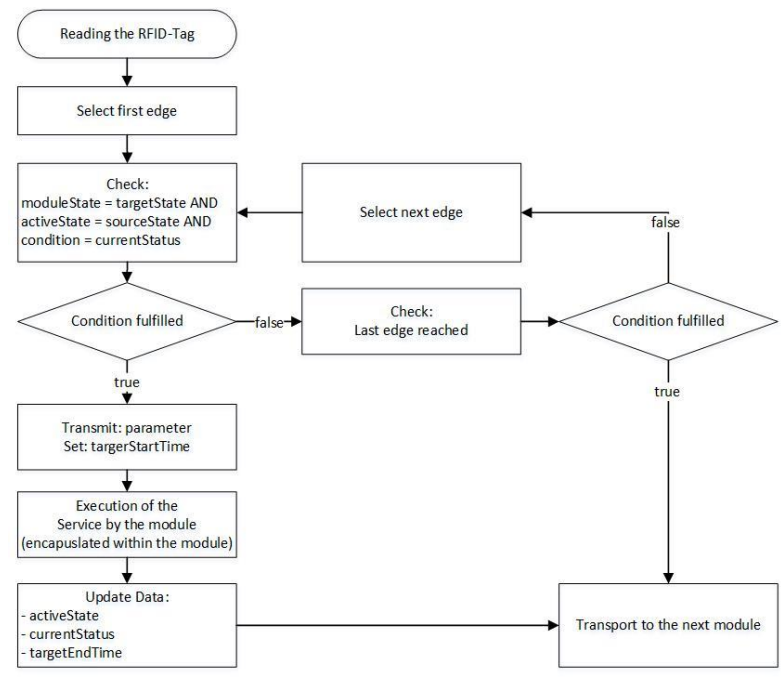

Fig. 3. Flowchart of the Processing of the Data

\section{$5 \quad$ Implementation}

The concept Automaton-on-Tag has been implemented in a modular manufacturing system, which is dedicated to the production of individualized cylinders. The RFID tags are integrated into the carriers of the cylinders. The modular manufacturing system consists of six independent production modules connected via a conveyor belt. Each module is equipped with an RFID reader to communicate with the RFID tags of the carriers that pass the module on the conveyor belt. The layout of the conveyor belt ensures that all carriers will pass every module as often as necessary. Due to the implementation of Automaton-on-tag the manufacturing system becomes more flexible: Each module just has to know which services it offers, the specific control code for the execution of the services is encapsulated within the module, hidden from the control of the production process. The implementation of the communication between the modules via the carriers results in a loose coupling of the modules and the separation from a centralized IT System. This enables a flexible adaption of the manufacturing system to dynamic changes by integrating or removing certain modules. The MES just has to know how many modules of each type are integrated to be able to release an optimal amount of production orders into the production process. Changes in the running production workflow can be done at any time without the consideration of several IT systems by adjusting the data on the RFID tags, e.g. by using a handheld RFID reader.

\section{Conclusion}

In this paper, we presented a concept for an RFID based production control in a modular manufacturing system. All necessary information is stored on an RFID tag, which is 
directly linked to the product. The interpretation of the data in form of a Mealy machine is suitable because it does not need much capacity on the RFID tag and enables a dynamic representation of complex production plans, including loops or alternative routes. The benefits of this concept are manifold: The whole system becomes more independent from a centralized backend system, making it far more resistant against disturbances and interruptions $[8,9]$. The manufacturing process becomes more flexible and changeable because the communication via RFID tags enables an easy addition or removal of modules. In addition, changes in a running workflow can be done easily because adjustments will be written directly onto the RFID tag related to a product. Further research questions which arise around MES in a loosely coupled modular plant are: How can an MES be aware of the modules and their services if it is not directly connected to each module? Future research might profit from the research around so called Module Type Packages (MTP). MTP are a promising way to describe the communication interfaces and functionalities of process plant modules [11].

\section{$7 \quad$ References}

1. Faltinski S, Henneke D, Jasperneite J (2014) M2M-Communication using RF-ID and a Digital Product Memory. In: Jahreskolloquium Kommunikation in der Automation (KommA 2014), Lemgo, pp 1-8.

2. Kagermann H, Wahlster W, Helbig J (2013) Umsetzungsempfehlungen für das Zukunftsprojekt Industrie 4.0. Technical Report, Arbeitskreis Industrie.

3. Fay A, Diedrich C, Thron M, Scholz A, Puntel-Schmidt P, Ladiges J, Holm T (2015) Wie bekommt Industrie 4.0 Bedeutung?. atp edition, 57: 30-43. doi: 10.17560/atp.v57i07-08.519

4. Schreiber S, Jerenz S, Fay A (2011) Anforderungen an Steuerungskonzepte für moderne Fertigungsanlagen. In: Tagungsband Automation, pp 28-29.

5. Dürkop L, Jasperneite J, Fay, A (2015) An analysis of real-time ethernets with regard to their automatic configuration. In: 2015 IEEE World Conference on Factory Communication Systems (WFCS), IEEE, pp 1-8. doi: 10.1109/WFCS.2015.7160548

6. Dürkop, L Trsek H, Otto J, Jasperneite J (2014) A field level architecture for reconfigurable real-time automation systems. In: 2014 10th IEEE Workshop on Factory Communication Systems (WFCS), IEEE, pp 1-10. doi: 10.1109/WFCS.2014.6837601

7. Wang C, Jiang P (2015) The approach of hybrid data on tag in decentralized control system. In: 2015 IEEE International Conference on Cyber Technology in Automation, Control, and Intelligent Systems (CYBER), pp 799-802, IEEE. doi: 10.1109/CYBER.2015.7288045

8. Hodek S, Floerchinger F (2009) An approach for modular production from mechanics to decentralized control, realized in the smartfactory KL. In: ETFA 2009. IEEE Conference on Emerging Technologies \& Factory Automation, IEEE, pp 1-7. doi: 10.1109/ETFA.2009.5347004

9. Nettsträter A, Nopper JR, Prasse C, ten Hompel M (2010) The internet of things in logistics. In: 2010 European Workshop on Smart objects: systems, technologies and applications (RFID Sys Tech), pp 1-8, VDE.

10. Lunze, J (2006) Ereignisdiskrete Systeme: Modellierung und Analyse dynamischer Systeme mit Automaten, Markovketten und Petrinetzen. Oldenbourg Verlag, Munich

11. Bernshausen J, Haller A, Holm T, Hoernicke M, Obst M, Ladiges J (2016) Namur Modul Type Package-Definition. atp edition, 58: 72-81. doi: 10.17560/atp.v58i01-02.554 